\title{
Sclerosant extravasation following endoscopic injection sclerotherapy for bleeding gastric varices
}

A 71-year-old woman was admitted to our hospital because of vomiting blood, and an endoscopic examination revealed ruptured gastric varices ( $\bullet$ Fig. 1). Endoscopic injection sclerotherapy was carried out by injecting a total of $2 \mathrm{~mL}$ of sclerosant (a mixture of $1 \mathrm{~mL}$ of cyanoacrylate and the same amount of lipiodol) into the varices ( $\mathbf{F i g . 2}$ ), and endoscopic hemostasis was achieved (๑ Fig. 3).

Immediately after the procedure, the patient complained of left upper quadrant pain, and she was treated with an analgesic. Radiograph and computed tomography ( $\odot$ Fig. 4 a,b) revealed a high-density area around the left diaphragm, and we made a diagnosis of extravasation of the sclerosant following sclerotherapy. The symptoms resolved 1 day after the procedure.

Only one previous report has focused on extravasation following sclerotherapy for bleeding gastric varices. Cheng et al. reported a case of intraperitoneal sclerosant leakage after sclerotherapy with a $3 \mathrm{~mL}$ mixture of cyanoacrylate and lipiodol [1]. Sclerosant extravasation following sclerotherapy for gastric varices is a very rare but serious complication.

Endoscopy_UCTN_Code_CPL_1AH_2AC

K. Akimoto, M. Inamori, T. Akiyama, H. Endo, K. Fujita, M. Yoneda, H. Takahashi, A. Goto, Y. Abe, H. Kirikoshi, N. Kobayashi, K. Kubota, S. Saito, N. Ueno, A. Nakajima

Gastroenterology Division, Yokohama City University School of Medicine, Japan

\section{References}

1 Cheng HC, Cheng PN, Tsai YM et al. Sclerosant extravasation as a complication of sclerosing endotherapy for bleeding gastric varices. Endoscopy 2004; 36: 239-241

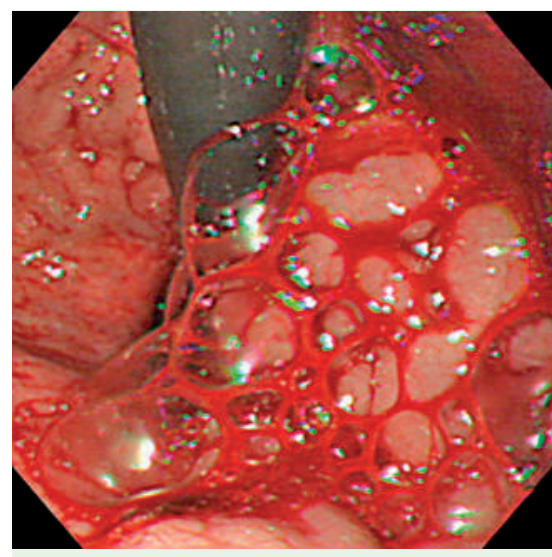

Fig. 1 Bleeding from gastric varices was revealed.

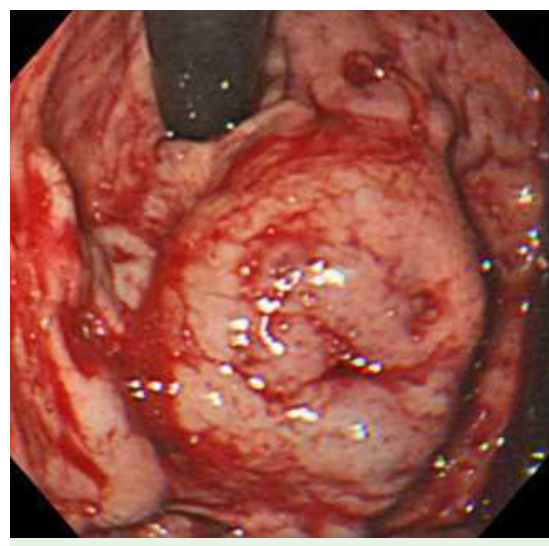

Fig. 3 Endoscopic hemostasis was successful.

\section{Bibliography}

Dol $10.1055 / s-2007-966832$

Endoscopy 2007; 39: E242

(c) Georg Thieme Verlag KG Stuttgart · New York . ISSN 0013-726X

\section{Corresponding author}

\section{Inamori, MD}

Gastroenterology Division

Yokohama City University School of Medicine 3-9 Fukuura

\section{Kanazawa-ku}

Yokohama 236-0004

Japan

Fax: +81-45-784-3546

Email: inamorim@med.yokohama-cu.ac.jp

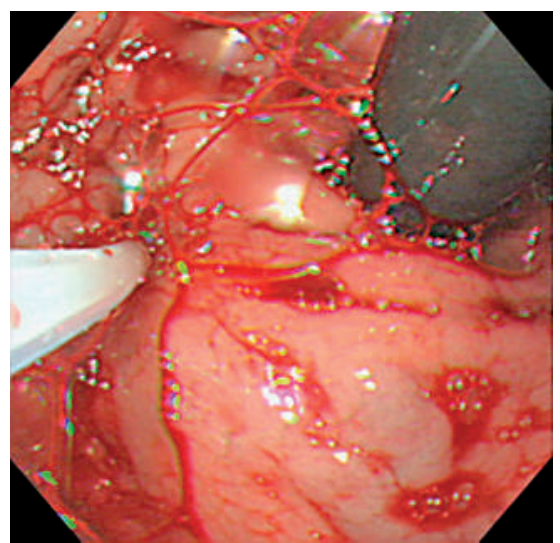

Fig. 2 Endoscopic injection sclerotherapy was carried out.
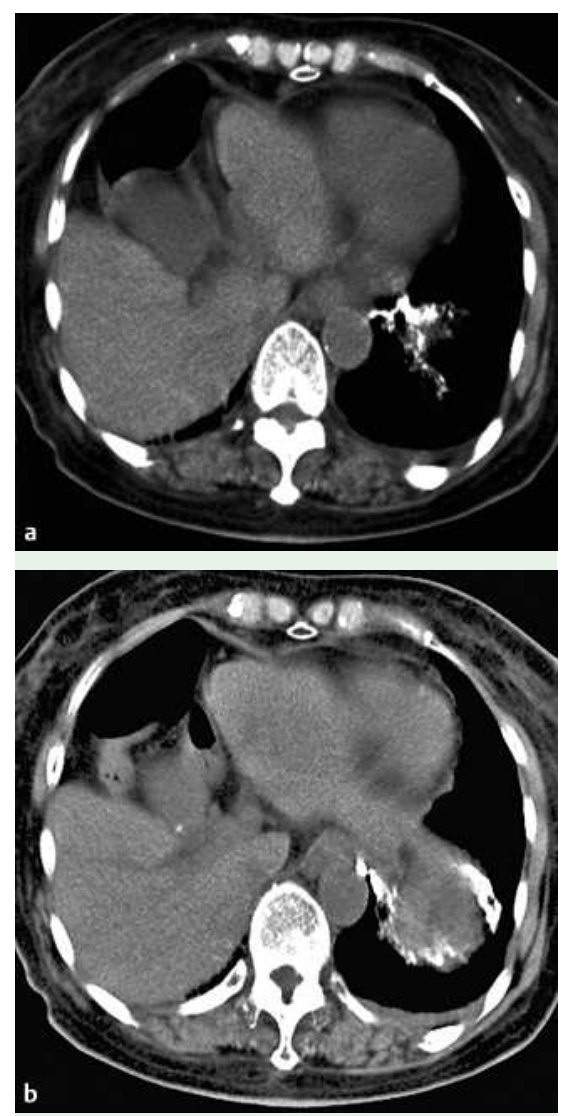

Fig. 4 a, b A computed tomography scan revealed a high-density area around the left diaphragm. 